\title{
Millimeter Wave Antennas
}

\author{
FELIX K. SCHWERING, FELLOW, IEEE
}

Invited Paper

\begin{abstract}
Major advances in mm-wave antennas have been made in recent years in particular in two areas. A new class of leakywave antennas based on open mm-waveguides has been proposed and investigated; and substantial progress has been achieved in integrated antennas where active and passive circuits, possibly in monolithic form, are combined with the radiating elements in one compact unit. Interesting developments have taken place also in a third group of mm-wave antennas, that of microstrip antennas and printed circuit antennas in general. An attempt is made to summarize these developments.
\end{abstract}

\section{INTRODUCTION}

Progress in the area of mm-wave antennas has been associated in recent years in particular with two groups of antennas, i.e., leaky-wave antennas derived from open mmwaveguides and integrated antennas. Both groups consist of radiating structures that essentially are new devices. While the antennas of the first group are based on a common principle, the perturbation of an open mm-waveguide such that it radiates in a controlled fashion as a leaky-wave line source, the second group includes a rather wide variety of radiating structures; the common feature is the use of solid-state devices integrated with the radiating elements in one compact unit that provides signal processing or control function in addition to acting as antenna. Some interesting developments have taken place also in a third area, that of printed circuit antennas, and in particular microstrip antennas, which are often used as the basic radiating elements in $\mathrm{mm}$-wave arrays. In the following an attempt is made to summarize the progress achieved in recent years in these three groups of mm-wave antennas.

We should point out, however, that some important advances have been made also in the area of mm-wave antennas of conventional design such as reflector and lens antennas. The available space does not allow to discuss these developments in any detail, but a few are mentioned here briefly as representative examples. A first case is recent work by Wiltse [1], [2] on Fresnel zone plate lenses which use diffraction, rather than refraction, to focus a mm-wave beam. The antennas combine good electrical performance with structural simplicity, an important design goal in the

Manuscript received November 3, 1990; revised March 4, 1991. The author is with U.S. Army CECOM, Fort Monmouth, NJ 07703. IEEE Log Number 9105089. mm-wave region. A second case is the development of lens shaping techniques for the $\mathrm{mm}$-wave region. Shaped lenses designed by Lee [3], [4] and Rotman [5] for multibeam operation in the $44-\mathrm{GHz}$ band have shown very good electrical performance combined with very reasonable dielectric weight, which would make these antennas excellent candidates for satellite applications. A third and fourth example are a geodesic dome antenna designed by Duford and Uyeda [6], which radiates a fan-shaped beam that can be scanned in the azimuth plane over a wide angular sector with very little beam degradation; and a holographic antenna designed by lizuka et al. [7] that uses a printed circuit board with a carefully designed metallization pattern to diffract a mm-wave beam radiated by a feed horn and, in the process, shape its radiation pattern.

These are examples. For a comprehensive review on mmwave antennas, that includes both existing and new designs, we refer to the literature and in particular to [8]-[12] which also contain extensive bibliographies on this subject.

\section{ANTENNAS DERIVED FROM OPEN AND Closed Millimeter Waveguides}

Most mm-waveguides such as dielectric guide and microstrip are open guiding structures, and energy leakage will occur when the uniformity of these guides is perturbed or they are not excited in the appropriate mode. This leakage effect may be used to advantage for the design of antennas, by intentionally introducing perturbations in these guides so that they radiate in a controlled fashion. A specific advantage of these antennas is their compatibility with the waveguides from which they are derived, thus facilitating integrated designs. The dielectric grating antennas of Fig. 1 belong to this class of radiating structures. Known for some time, they have found renewed interest as mm-wave antennas and have been studied systematically only recently and in the mm-wave context [13]-[17]. These periodic antennas are leaky-wave line sources whose radiation direction includes an angle with the forward direction. The radiation angle varies with frequency and the antennas can be used for frequency scanning.

Figure 2 shows a group of new open waveguide $\mathrm{mm}$ wave antennas that have been suggested in recent years by Oliner. Similar to the antennas of Fig. 1 they operate 


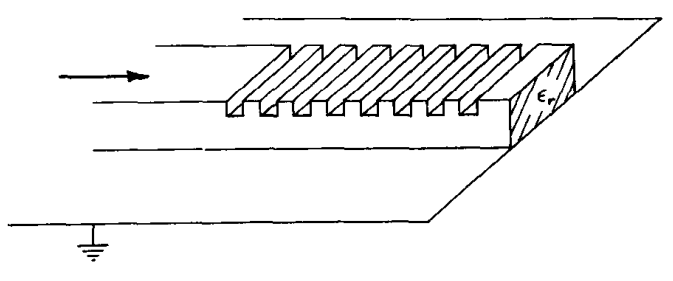

(a)

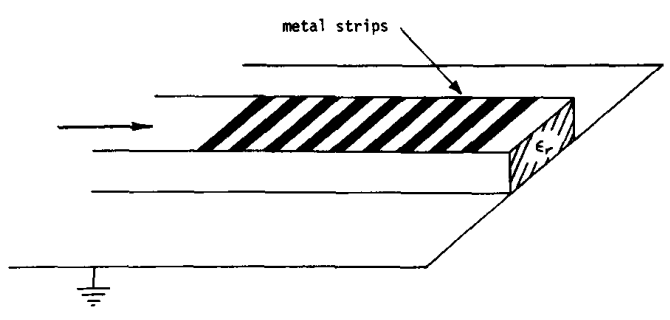

(b)

Fig. 1. Periodic dielectric antennas. (a) With dielectric grating. (b) With metal grating.

basically as leaky-wave line sources that scan with frequency. But they use methods other than periodicity to produce radiation and are uniform, rather than periodic, in the longitudinal direction; they may be called "uniformwaveguide leaky-wave antennas." These antennas have been studied systematically during the past few years by Oliner, in cooperation with Lampariello, Shigesawa, and their associates, and can be regarded as well understood by now; see in particular a comprehensive recent report by Oliner [18] where the theory of these antennas is presented together with detailed design information. A bibliography on the antennas may be found in this report [18] and in [8] and[9].

Some $\mathrm{mm}$-waveguides, notably metal waveguide and finline, are closed rather than open guiding structures and the design of antennas that are directly compatible with these waveguides takes a somewhat different approach. The printed tapered slot antennas recently investigated by the Yngvesson group [19], [20] are well suited for this purpose [9]. Figure 3 shows three versions of these antennas. They are traveling wave devices that, similar to surface wave antennas, radiate in the end fire direction; their planar configuration allows fabrication by photolithographic methods.

\section{A. Periodic Dielectric Antennas}

These antennas [13]-[16] consist of a uniform dielectric waveguide with a periodic surface perturbation; see Fig. 1. The waveguide is excited in the fundamental mode and its width is usually chosen comparatively small to guard against the excitation of higher order modes. The surface perturbation of the waveguide may take the form of a dielectric grating, Fig. 1(a), or one may use a grating of metal strips, Fig. 1(b). Diffraction at this grating will transform the basic guided mode into a leaky wave and
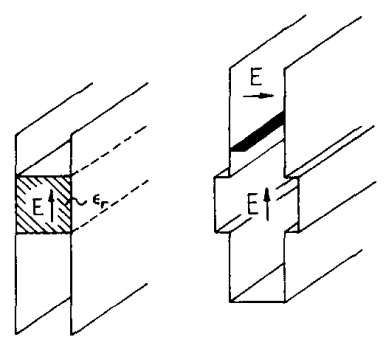

(a)
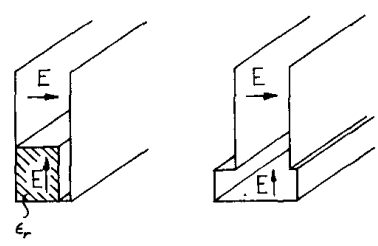

(b)

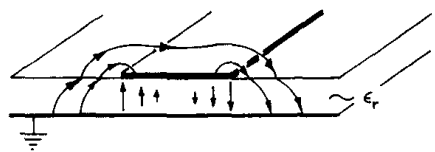

(c)

Fig. 2. Uniform waveguide leaky-wave antennas. (a) NRD guide antenna using foreshortened upper waveguide arm and groove-guide antenna using asymmetric metal strip. (b) NRD guide antenna using asymmetric air gap and groove-guide antenna using off set waveguide stub. Both antennas have been bisected horizontally relative to their unperturbed waveguides and closed off at the bottom. (c) Microstrip traveling wave antenna using first higher mode in its leaky wave regime (after Oliner [9], [18])

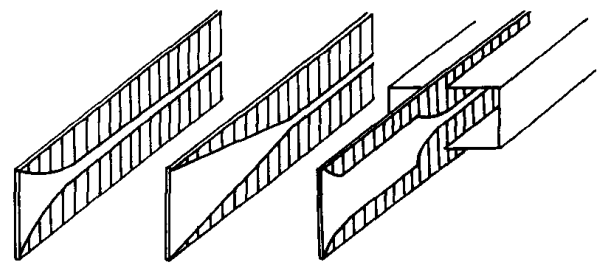

(a)

(b)

(c)

Fig. 3. Printed tapered slot antennas: (a) Vivaldi antenna (exponential taper). (b) Linear taper. (c) Antenna with constant width section (after Yngvesson et al. [19]).

the waveguide becomes an antenna. As the frequency is increased, the main beam direction scans from backfire through broadside and into the forward quadrant, even reaching endfire if the parameters are chosen appropriately.

The scan rate of these antennas tends to increase with the permittivity of the antenna material ${ }^{1}$ and is typically in the range of $20^{\circ}-40^{\circ}$ for a $10 \%$ frequency variation [8],

${ }^{1}$ A periodic antenna made from ferrite (rather than dielectric) material may be scanned at constant frequency by varying the biasing magnetic field. An antenna of this type has been investigated by Maheri, et al. [17]. 
[9], [13]. Note, however, that these grating antennas, as all periodic antennas, do not radiate in the exact broadside direction, where an internal resonance inhibits radiation. The associated "stopband," however, is narrow for typical antennas and the only effect usually noticed when scanning through broadside is an increase in SWR.

\section{B. Uniform Waveguide Leaky-Wave Antennas}

The antennas sketched in Fig. 2 are based on other open waveguides [9], [18], specifically groove guide, NRD guide (which is a recently studied variant of $\mathrm{H}$ guide) and microstrip line. As opposed to periodic antennas, these devices have the outward appearance of uniform open waveguides, but they are leaky and act as antennas.

To explain the operating principle [9], [18] of the first two antennas, Fig. 2(a), we note that the electric field of the fundamental mode of the corresponding unperturbed guides is essentially vertically polarized. The central region of these guides is wider than the regions above and below, physically in the groove guide and electrically in the NRD guide, and operates above cutoff transversely for the fundamental mode, which is guided primarily in this region. The two waveguide arms above and below the central region have a smaller width and operate below cutoff transversely so that the fundamental mode decreases exponentially away from the central region. If these waveguide arms are sufficiently long, they may remain open at the ends; no energy will escape.

In the case of the NRD guide antenna, radiation is obtained by foreshortening the upper waveguide arm; the leakage constant can be sensitively controlled by the remaining height of this upper arm. In the case of the groove guide antenna, a small metal strip is placed in an asymmetric fashion in the (unshortened) upper arm. Diffraction of the fundamental mode at this strip will produce a cross-polarized perturbation field due to the asymmetry. Being polarized horizontally, this field does not have a cutoff frequency and will travel, in the fashion of a TEM parallel-plate-guide mode, to the open end of the waveguide arm where it is radiated out. The direction of radiation of both the NRD guide antenna and the groove guide antenna varies with frequency, but, as opposed to periodic antennas, the scan range is confined to the forward quadrant only. This limitation is due to the fact that the antennas are uniform in the longitudinal direction; it holds for all the antennas in Fig. 2. While periodic antennas have the greater design versatility, the antennas in Fig. 2 have the advantage of greater structural simplicity.

The antennas in Fig. 2(b) are also derived from NRD guide and groove guide [9], [18], but both antennas use asymmetry in the guide cross section to produce radiation. In addition, relative to the waveguides from which they are derived, these antennas have been bisected horizontally to obtain a simpler structure and provide radiation from one side only.

The antenna of Fig. 2(c), finally, is based on microstrip and a different method is used to produce radiation [9], [18], [21]. The guiding structure in this case is not perturbed, so that the fundamental mode remains a purely bound wave. Instead, the microstrip is excited in its first higher mode which is leaky in a frequency band just above cutoff. The line dimensions are then chosen such that the first higher mode is within its leaky wave regime, and by an appropriate feed arrangement it is insured that only this mode is excited. A particular advantage of the antenna is its remarkable structural simplicity.

A very useful feature of the antennas of Figs. 1 and 2 is that they are well suited for pattern shaping [8], [9], [18], [22]. A certain exception is the microstrip leaky wave antenna of Fig. 2(c) whose structural simplicity also limits its versatility. But for several of the other antennas, accurate pattern shaping techniques have been developed with the added advantage that they are easily implemented [18], [22].

\section{Arrays of Leaky-Wave Antennas}

The axial length of the leaky-wave antennas of Figs. 1 and 2 is typically 10 to $50 \lambda_{o}$, i.e., the antennas are long in the forward direction and their beamwidth in the principal plane parallel to the longitudinal axis is narrow. The lateral width of these antennas, on the other hand, is small and their beamwidth in the plane normal to the longitudinal axis wide. Pencil beams with a narrow beamwidth in both principal planes may be achieved by the use of an array of several of these line source antennas in a parallel arrangement.

Figure 4 is a cross section view of two such arrays, consisting of antennas of the type shown in Fig. 2(b), which leak because of asymmetry [9], [18]. It is assumed here that each of the line sources is fed from one end with an arrangement in which a phase shift can be introduced between successive line sources. The array can then be scanned in the longitudinal plane by frequency variation and in the cross plane by phasing. The vertical metal baffles [9], [18] separating the element antennas serve to eliminate blind spots, a major problem that usually arises in arrays of this type, and they also allow to place the line sources directly next to each other with minimal mutual interaction. Since each line source is less than $\lambda_{o} / 2$ wide, no grating lobes will be encountered during the scans, and crosspolarized radiation will be suppressed provided the baffles are high enough $\left(>\lambda_{o} / 2\right)$ so that the mode with vertical polarization, which is below cutoff in the air-filled region, has decayed sufficiently at the aperture.

Arrays of this type are structurally simple, low-profile antennas that provide design versatility in addition to their excellent electrical performance. They should be well suited for a variety of mm-wave applications. The design of feed systems that match the structural simplicity and electrical performance of the arrays will require special care, however. The scan range of the arrays of Fig. 4 is limited to the forward half of the upper hemisphere but can be enlarged [9], [18] to cover the backward half as well if the longitudinally uniform line sources are replaced by periodic antennas of the type shown in Fig. 1. 


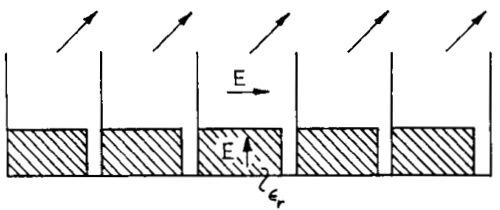

(a)

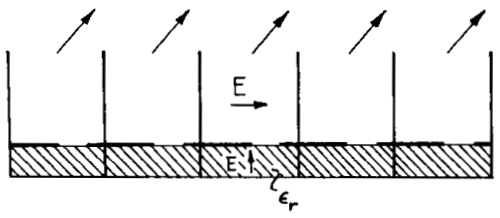

(b)

Fig. 4. Arrays of leaky-wave line sources that can be scanned in one principal plane by frequency variation and the other by phasing. The vertical metal baffles permit these arrays to have high polarization purity, no blind spots and no grating lobes. (a) Array of NRD guide antennas with asymmetric air gaps. (b) Array of printed-circuit groove-guide antennas with off set apertures (after Oliner [18]).

\section{Tapered Slot Antennas}

Typically these antennas (Fig. 3) are printed on a thin substrate and their axial length is 3 to $12 \lambda_{o}$. Correctly designed tapered slots provide good electrical performance, including a moderately high gain of $10-17 \mathrm{~dB}$, relatively low side lobes, a circular symmetric main beam, and practically constant impedance over a broad frequency band [19]. Achieving circular polarization or dual polarization, however, is a problem that is not easily solved.

The Yngvesson group has studied these antennas in particular for array applications [19], [20]. Since tapered slot antennas are endfire devices, their directivity gain is primarily determined by their axial length while their cross section width is small. Hence, when used as array elements, they can be packaged closely. Although this will reduce their directivity, their port-to-port isolation tends to remain fairly high. Since, in addition, these antennas are amenable to integration of monolithic solid-state devices such as Schottky diodes or SIS mixers, and their depth dimension provides ample room for integrated circuits, they are well suited for the design of feed arrays for high resolution mm-wave imaging systems and multibeam satellite communication systems [19]. Other applications include scanned arrays for tracking systems, and integrated arrays for quasi-optical power combining [20].

\section{Microstrip ANTENNAS AND Other PRINTED CiRCUIT ANTENNAS}

Printed circuit antennas are simple in structure and easy to fabricate by lithography. They are low profile, light weight, low cost devices that are well suited as radiating elements for planar and conformal array antennas. In active arrays they allow convenient integration with active and passive circuits for beam control and signal processing.
The best known printed circuit antennas are microstrip patch resonator antennas and microstrip dipole antennas. These antennas have been studied extensively during the past ten or twenty years, at microwave frequencies, and can be regarded as well understood by now. The only problem with microstrip antennas, and in particular patch resonator antennas, is a narrow bandwidth which would not allow their use in broad-band systems. For a general review on microstrip antennas we refer to the paper by Pozar in this special issue, which also discusses broadbanding techniques and the important question of the design of appropriate feed systems for phased arrays of these antennas, including their integrated versions. The present discussion addresses their mm-wave aspects and is concerned primarily with passive microstrip antennas and arrays. Active versions are discussed in the section on integrated antennas.

The extension of microstrip antennas into the mm-wave region is not simply a matter of straightforward wavelength scaling. New problems as well as new opportunities appear. There are in particular two problems: fabrication tolerance and feedline losses. Both problems are associated primarily with the feed systems of mm-wave microstrip arrays rather than with the radiating elements themselves. For ease of fabrication, the feed system, in microstrip, is usually printed with the radiating elements on the same substrate surface [23], or it may be embedded in the substrate [24]. Typically the feedlines have a width in the order of a few tenths of a millimeter for an array operating at a frequency in the $30-100 \mathrm{GHz}$ band, and required tolerances are tighter by an order of magnitude. Thus highly precise fabrication techniques are needed [23]. Furthermore, microstrip lines are not low loss lines, and in large arrays, which are needed to obtain a high directivity gain, feedline losses can be substantial, in particular when a corporate feed system is used.

Various methods have been studied in recent years to resolve these problems and in particular the efficiency problem [8], [9], [23]-[26]. The results obtained lead to the conclusion that microstrip patch resonator arrays will be useful antennas for the lower mm-wave band up to a frequency of $100 \mathrm{GHz}$ and possibly $140 \mathrm{GHz}$ [8], [26]. For this band it should be possible to design microstrip antenna arrays of fairly high gain that provide good pattern quality, low SWR over a bandwidth of several percent, and acceptable efficiency [23], [26], [27].

An advantage of microstrip mm-wave antennas is that their bandwidth limitation can be overcome by the use of electrically thick substrates. In the microwave region, the substrate thickness is typically only a small fraction of a wavelength. An antenna printed on a thin, grounded substrate will have to operate in close proximity to the ground plane, which implies the use of resonator type antennas of high $Q$ in order to raise the radiation resistance to reasonable values. This, in turn, leads to a narrow bandwidth. In the mm-wave region, on the other hand, a substrate that is physically thin can still have an electrical thickness of $\lambda / 4$ or more. Hence, there is no need for 
the use of high- $Q$ resonator antennas, but one can use other antenna configurations which provide much broader bandwidths as, for example, printed dipole antennas of large width. The use of thick substrates has the additional advantage that fabrication tolerances become less critical.

A problem with thick substrates is the trapping of surface waves. These waves will carry power away from the antennas but do not contribute to the (desired) radiation pattern so that their power must be accounted for as antenna loss and the efficiency decreases. In addition, pattern distortions will occur since, eventually, these surface waves will radiate, in a parasitic fashion, when they reach the end of the substrate or any other obstruction in their propagation path. The design problem is to obtain a substantially enhanced bandwidth while maintaining good efficiency. This problem has been investigated a few years ago by the Alexopoulos group [28] for the case of printed linear dipole antennas. An independent investigation by Pozar [29] has led to results in good agreement with the Alexopoulos study. The study has shown [28] that, for optimum performance, the substrate height should be about a quarter wavelength in the substrate material and that the permittivity of the substrate should not be too high; $\epsilon_{\mathfrak{r}}$ should be 4.5 or smaller. $^{2}$

In this case, the radiation efficiency will be better than $50 \%$, i.e., more than $50 \%$ of the available power will be radiated and less than $50 \%$ trapped in surface waves (with the ratio improving with decreasing $\epsilon_{r}$ ), and a maximum bandwidth is obtained which for a thin wire dipole is $18 \%$, a substantial improvement over the bandwidth of microstrip patch resonator antennas on thin substrates that is in the order of a few percent only. For dipoles of large width, numerical data are not as yet available. But it can be expected that their bandwidth will noticeably exceed the $18 \%$ value attainable with linear dipoles. The bandwidth referred to here is the impedance bandwidth.

A common requirement on mm-wave antennas is a high directivity which implies the use of arrays of microstrip dipole antennas on thick substrates. The design of such arrays involves new problems. Surface waves trapped in the substrate are likely to increase mutual coupling between the array elements. A low-loss feed system is needed that is easy to fabricate but keeps feed line radiation to a minimum. And in the case of a scanned array, the occurrence of blind spots may severely limit the useful scan range. These problems are not easy to solve. A promising approach to the feed system problem is the use of a two-layer substrate, as investigated by Katehi and Alexopoulos [32]. Here the feedlines, in microstrip, are printed on the lower layer which is electrically thin so that the energy guided by the feedlines is tightly bound and little feed system radiation will occur. The (unbroken) dipoles are printed on the upper

\footnotetext{
${ }^{2}$ Hence, materials such as teflon, polystyrene, and quartz would be useful substrates while $\mathrm{Si}$ or GaAs $\left(\epsilon_{r} \approx 12\right)$, which are needed for integrated antennas, would lead to poor efficiency at the substrate heights considered here. A way out of this problem is the use of a very thin Si or GaAs layer (in which any monolithic components can be created) which is bonded to a quartz substrate that satisfies the above condition. This approach has been used, for example, by Rutledge [30] and Fetterman [31].
}

layer and are excited by near-field coupling without the need for conductive contact to the feedlines. In the case of a thick substrate, as assumed here, it may be necessary to enhance this coupling with the help of parasitic dipoles [33] embedded in the upper substrate layer or printed with the radiating dipoles on the top substrate surface. The use of such dipoles has the added benefit that it will increase the bandwidth of the antenna.

A recent theoretical investigation by the Alexopoulos group [34]-[37] has shown furthermore that the performance of microstrip dipole antennas (on thin or thick substrates) can be improved significantly by the use of a superstrate, i.e. by covering the printed dipole by an additional dielectric or magnetic layer where the permittivity or permeability of the superstrate should exceed that of the substrate. By careful parameter selection, surface waves can be suppressed almost completely [34], resulting in a radiation efficiency close to $100 \%$ over a narrow bandwidth. Alternatively, improvements in radiation resistance and pattern shape may be obtained over a relatively broad bandwidth [34], [35]; or as a somewhat surprising result, a very high directivity gain can be achieved with a single dipole [36]. This high gain effect has been explained by Jackson and Oliner [37] in terms of a radially propagating leaky wave of low leakage constant, that is supported by the substrate-superstrate configuration. The effect implies the use of a superstrate that consists of several layers whose permittivities (permeabilities) alternate between a high and a low value, while the electrical thickness of each layer is a quarter wavelength at the design frequency. At this frequency the gain achieves its maximum and the pattern takes the shape of a narrow, broadside directed main beam without side lobes, a pattern shape ideal for many applications. The pattern bandwidth however is very narrow.

\section{INTEGRATED ANTENNAS}

The term integrated antennas is used for a class of radiating structures where devices such as solid-state oscillators, detectors, phase shifters and filters are integrated with the radiating elements on the same substrate, typically in monolithic form. The substrate may consist of several layers if more complex functions have to be accommodated.

Integrated antennas have the obvious advantages of compactness, reliability, reproducibility and, if fabricated in large numbers, of low cost. These antennas should be useful not only for the lower mm-wave region but in particular also for the upper mm-wave region above $100 \mathrm{GHz}$; and it can be expected that eventually they will be extended into the submillimeter-wave region as well. The small size of radiating elements, circuits, and components in these frequency bands strongly suggests the use of integrated and monolithic designs.

A considerable number of integrated mm-wave antennas has been developed in recent years for a variety of applications, and it would be difficult to give here a comprehensive review on these antennas. Instead, an attempt is made to 
indicate trends by subdividing integrated antennas into four groups and discussing a few representative developments in each of these groups (with the exception of the fourth group which is the subject of a different paper of this Special Issue). For information on other integrated antennas we refer to the literature [10] and in particular to the excellent review articles written by Itoh [38] and Rutledge et al. [39].

A first group of integrated mm-wave antennas consists of receiving antennas with integrated mixers or detectors. A relatively large number of these antennas has been studied in recent years and, though the development of most of these devices is still in the laboratory phase, their technology is fairly mature [38], much more so than for the antennas of the three remaining groups. A second group is formed by transmit antennas with integrated solid-state sources where the radiating structure serves simultaneously as resonator for the sources. A third group consists of integrated antennas which are designed for other functions and do not necessarily fit into the first two groups. As examples we will discuss an electronically controlled beam steering reflector and a frequency multiplying slot array. A fourth group, finally, are integrated phased array antennas where phase shifters, with their control circuits, and power amplifiers (transmit case) or low noise amplifiers (receive case) are integrated with the radiating element in one functional unit. From the applications viewpoint these integrated arrays are a very important class of (future) mm-wave antennas, and much of the work on the integrated receiveand-transmit antennas of the first and second group is driven by the need for integrated phased arrays. At the small dimensions in the mm-wave region, wafer-scale integration becomes feasible (replacing the modular design approach typically used in the microwave region) and an entire phased array antenna of fairly high gain can, in principle, be fabricated on a single "chip." The substrate may have to consist of several layers, however, in order to accommodate the various functions of the array, and the array architecture becomes and important design consideration. Pioneering work on these arrays is in progress at industry, in particular on array technology for the 20,30 , and $44-\mathrm{GHz}$ bands [40], [41], and this work is currently extended to $60 \mathrm{GHz}$ [42]. These developments are reviewed in the article, "Antennas array architecture," by Mailloux in this special issue and the discussion in the present paper is limited to the first three groups of mm-wave antennas.

\section{A. Receive Antennas with Integrated Detectors}

These antennas combine the function of a receive antenna and a mixer or detector in one device. The output terminals of the antenna and input terminals of the mixer are in close proximity to each other and usually not accessible from the outside. Hence, the RF path between the antenna and the mixer is very short, or completely eliminated, and the noise figure of these devices is expected to be superior to that of an antenna and mixer system of conventional design [38]. The local oscillator (LO) signal may be coupled into the mixer through a guide of some kind or it may be received quasi-optically through the antenna.

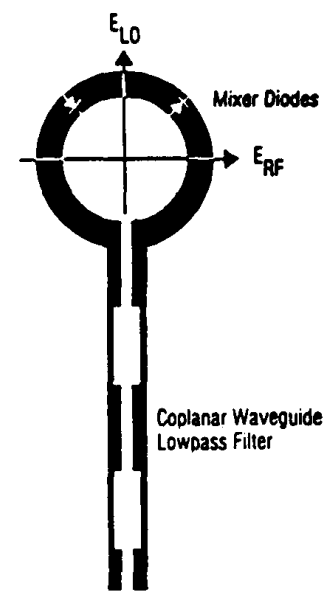

To If Ampltier

Fig. 5. Ring slot antenna with integrated mixer diodes. The ring slot, shown in black, is etched into the one-sided metal cladding of a dielectric substrate. Arrows indicate RF and LO field polarizations (from Itoh [38]).

A simple but instructive device of this type is shown in Fig. 5. Designed by Stephan, Itoh, and Camilleri [38], [43], the antenna consists of a circular slot etched into the metal cladding covering one side of a substrate. At two positions off set from the vertical axis by $\pm 45^{\circ}$, the slot is bridged by balanced mixer diodes. The RF signal and the LO signal are assumed to be incident in directions perpendicular to the antenna plane, the RF signal at horizontal polarization from the front, the LO signal at vertical polarization from the rear. The LO signal drives the diodes so that the RF signal is down-converted at each diode. The resulting IF signals are combined and leave the antenna through a low pass filter built into the coplanar output waveguide. Different versions of the device have been designed for frequencies up to $35 \mathrm{GHz}$.

A second integrated receive antenna is shown in Fig. 6 . The antenna operates at $94 \mathrm{GHz}$ and was designed by the Rutledge group as an imaging array for plasma diagnostics [30], [44]. Its basic structure consists of a linear array of parallel dipoles, each with its own detector, that are printed on a thin GaAs wafer; the wafer is supported by a quartz substrate which serves as the base plate of the array. Typically the antenna would be placed as a receiving array into the focal plane of a large lens or reflector antenna. The multidetector approach overcomes the limited sensitivity and speed of conventional imaging systems using a single, mechanically scanned detector. Image resolution approaches the diffraction limit [30], [44].

Figure 6(a) shows some of the dipoles of the array. They are bow-tie dipoles that provide a reasonably broad-band impedance match to the detector diodes at their feed points. The detectors are Schottky diodes which are monolithically implanted in the GaAs wafer. The extension arms of the dipoles serve as low frequency leads for the diode currents. 


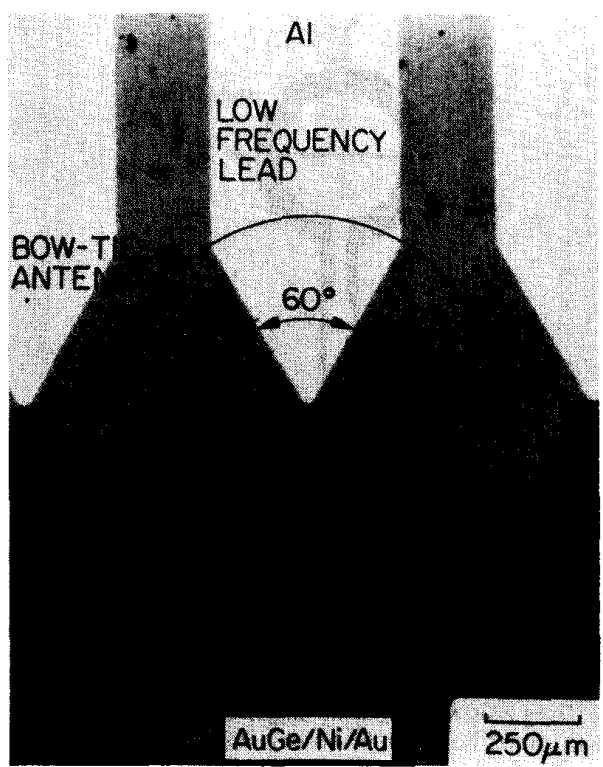

(a)

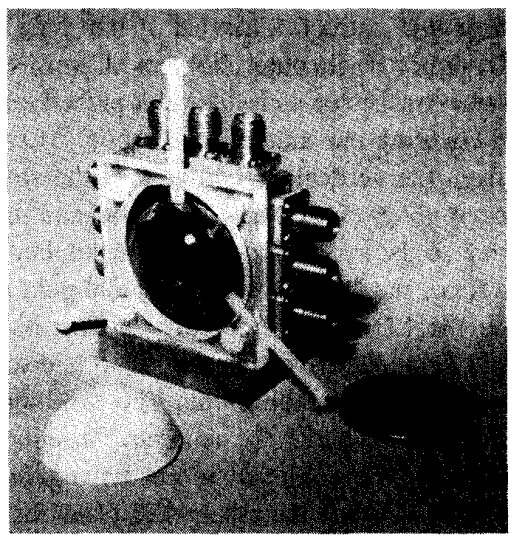

(b)

Fig. 6. Imaging array with integrated detector diodes designed for $94-\mathrm{GHz}$ band. (a) Array elements (printed bow-tie dipoles) with integrated detector diodes. (b) Finished device with substrate lens (courtesy Rutledge, from Zah et al. [44]).

The width of a 9-element dipole array is about $0.7 \mathrm{~cm}$ at $94 \mathrm{GHz}$.

Originally, microbolometers were used as detectors which resulted in the better noise performance. The use of Schottky diodes has the advantage that it permits heterodyning and leads to superior sensitivity [30], [39]. The finished device, Fig. 6(b), includes a substrate lens, to eliminate the conditions under which surface waves could be trapped in the (thick) quartz ground plate.

A two-dimensional array of integrated antennas [45] was also designed by the Rutledge group and is further developed by Rebeiz, a former member of this group. As shown in Fig. 7(a) the array consists of dipole antennas with integrated microbolometers, which are suspended in pyramidal horns. The horn array is etched into a silicon wafer structure with a minimum of two stacked wafers as indicated in Fig. 7(b). The dipoles and microbolometers are printed, by standard photo lithographic techniques, on a thin $(1-\mu \mathrm{m})$ silicon-oxynitrite membrane which is placed between the two wafers of the horn array. The space on the membrane between the horn apertures provides ample room for low frequency connections and processing electronics. The size of the horn apertures and the period of the array is typically 1.0 to $1.5 \lambda_{o}$. The horns are fabricated by anisotropic etching of silicon wafers using a solution that naturally forms pyramidal holes bounded by $<111>$ crystal planes in $\langle 100\rangle$ silicon. The aperture angle of the horns is fixed at $70.6^{\circ}$ by the orientation of the crystal planes and is somewhat larger than desirable from the electrical viewpoint. Smaller aperture angles may be obtained by the use of more expensive fabrication methods [45].

Models of the array have been developed for the 94$\mathrm{GHz}$ band and several other frequencies in the upper mm-wave region. The technology seems well suited for extension into the terahertz region as well [45]. Allowing effective diffraction-limited imaging, the arrays should be useful for remote sensing, plasma diagnostics, radiometry, and low-noise radio astronomy receivers. A dual polarized version suitable for polarimetric measurements at $94 \mathrm{GHz}$ is currently under development [46]. A key component is a novel orthogonal two-dipole feed in printed technology.

\section{B. Transmit Antennas with Integrated Oscillators}

A monolithic $44-\mathrm{GHz}$ transmit antenna with a pair of integrated IMPATT diodes has recently been developed by Camilleri and Bayraktaroglu [47]. The configuration of the device is shown in Fig. 8(a). Both, the pair of IMPATT diodes and the antenna circuits, are produced on the top surface of a semi-insulating GaAs wafer with a typical thickness of $100 \mu \mathrm{m}$. Via holes are used to ground one terminal of each diode. The radiating element consists of a section of microstrip line, one half guide wavelength long, that is placed between the two diodes and connected to these diodes by air bridges. The ground plane provides the return path and the antenna operates as a microstrip loop antenna that simultaneously serves as resonator for the diodes, determining their frequency of operation.

Typical results of output power and efficiency measurements for a 43.3-GHz device are shown in Fig. 8(b). For convenience, the measurements were conducted in waveguide circuits. The physical size of the antenna is small; the antenna chip measures $1.25 \mathrm{~mm} \times 0.75 \mathrm{~mm}$.

An integrated transmit antenna investigated by Birkeland and Itoh [38], [48], is based on a similar concept but uses a different implementation. The device is sketched in Fig. 9. Also this antenna utilizes a planar radiating structure that, at the same time, serves as resonator for an integrated solid-state oscillator. But the radiating structure is a periodic antenna in the form of a linear, series-fed microstrip patch antenna array and the oscillator is a negative resistance FET element. In general, FET's show a higher dc-to-RF conversion efficiency than diode sources [48]. Instead of the 


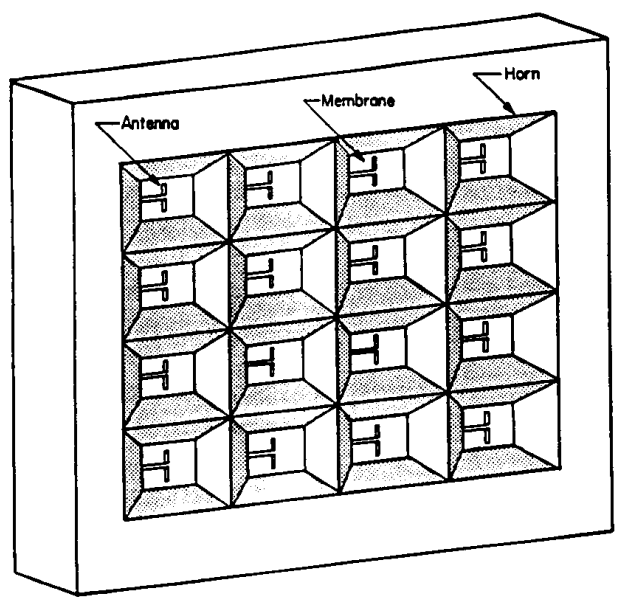

(a)

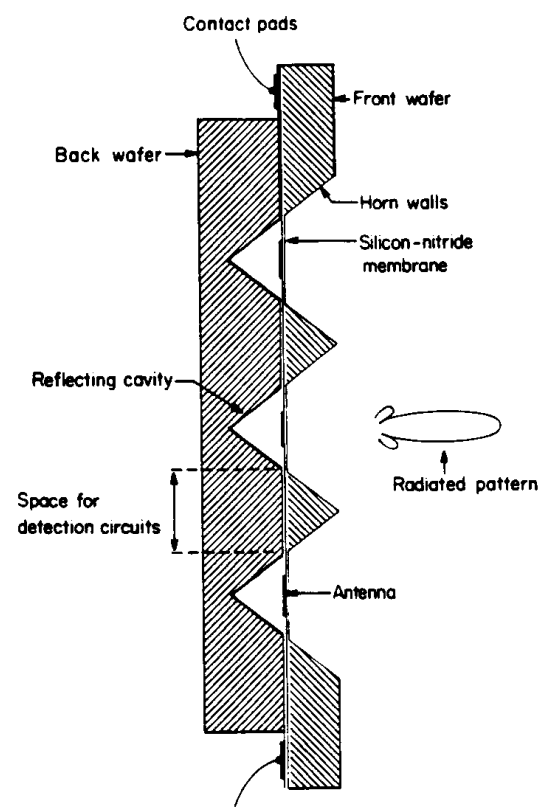

(b)

Fig. 7. Two-dimensional imaging array using printed dipoles with integrated microbolometers suspended in pyramidal horns. The horns are etched from silicon wafers. (a) Perspective view. (b) Cross section view of two-wafer structure (from Rebeiz et al. [45]).

microstrip patch antenna array employed here, a periodic dielectric antenna of the type shown in Fig. 1 could be used as an alternative. Such antennas, utilizing an integrated Gunn diode as oscillator, were studied earlier by Song and Itoh [49].

As explained in Section II, periodic antennas are leaky wave devices whose radiation direction scans with frequency. The radiation angle may be in the forward or backward quadrant. But, as broadside conditions are approached, i.e., as the guide wavelength approaches the

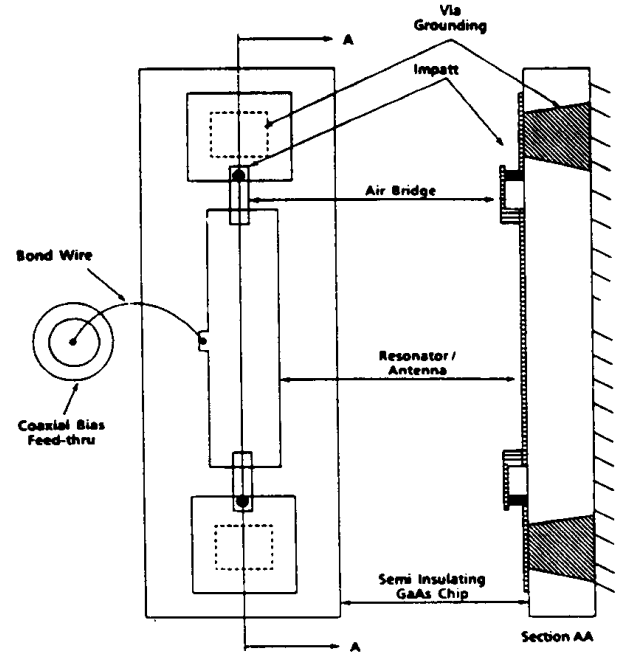

(a)

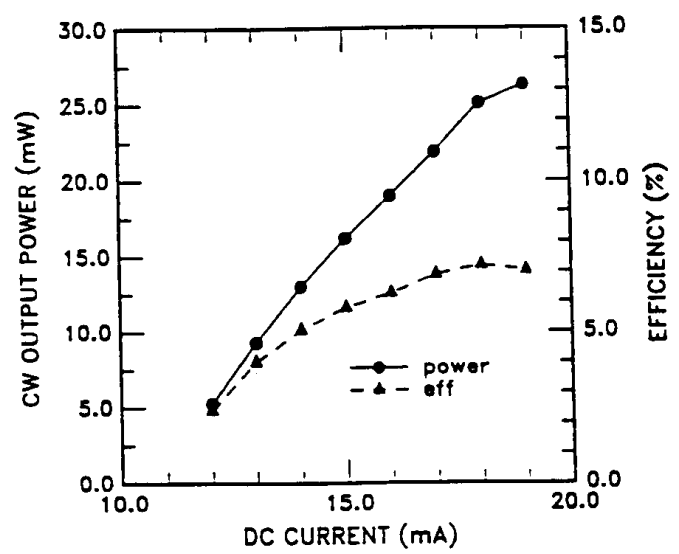

(b)

Fig. 8. Monolithic mm-wave transmit antenna with pair of integrated IMPATT diodes. (a) Antenna and resonator structure. (b) Output characteristics of $43.3-\mathrm{GHz}$ device measured in waveguide (from Camilleri and Bayraktaroglu [47]).

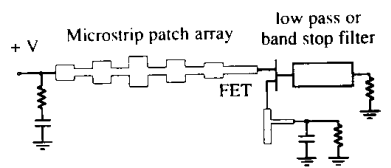

Fig. 9. Periodic microstrip patch array with integrated FET oscillator (from Birkeland and Itoh [48]).

period of the antenna, a stopband is encountered that leads to a high standing wave ratio (SWR) and, for passive antennas, inhibits radiation. In the active antenna considered here, the high SWR of the stopband is used to advantage to resonate the FET which will act as an oscillator operating at the stopband frequency. The power output of the FET is delivered to the antenna which now radiates in the broadside 
direction. The gate of the integrated FET is terminated in a bandstop or low-pass filter which provides the correct reactance at the oscillation frequency, while it prevents undesired oscillation in a lower (surface wave) stopband.

The antenna was demonstrated at $X$ band frequencies. Extension into the mm-wave region should not be a problem since the device is rather simple in structure [48]. Furthermore, as shown by Birkeland and Itoh [38], [48], the antenna requires only a slight modification for use as transceiver circuit, where the FET performs a dual function, serving as the source for the transmitted signal and as selfoscillating mixer for the down conversion of the received signal.

A completely different approach to the design of integrated mm-wave transmit antennas is the use of quasioptical power combiners of the kind investigated by Mink [50]. As indicated in Fig. 10, these combiners employ an open resonator of the Fabry-Perot type to injection lock an array of integrated solid-state sources distributed over the surface of one of the reflectors; a high- $Q$ resonant mode excited in the resonator forces these sources to operate in phase and frequency synchronism. The second reflector is partially transparent and the combined power is extracted through this surface in the form of a coherent wavebeam. A particular advantage of these quasi-optical combiners is that they may have dimensions of many wavelengths and may accommodate numerous sources [10], [50].

Evidently, power combiners of this type can readily be used as integrated transmit antennas provided that an appropriate modulation technique for the sources is devised. Beam shaping is conveniently achieved by choosing an appropriate geometrical shape of the reflectors, though the relationship between beam profile and reflector shape is not a simple one. The resonator investigated by Mink uses a planar and a uniformly curved reflector and produces a radiation field with a narrow Gaussian mainbeam and very low side lobe, i.e., a radiation pattern that is ideal for many applications. A combiner of this type has been demonstrated at $40 \mathrm{GHz}$. Quasi-optical power combiners employing Fabry-Perot resonators with two planar reflectors have been investigated at microwave frequencies by Compton and York [51], [53] and by the Rutledge group [52], [53]. The very simple resonator geometry makes these combiners particularly well suited for extension into the mm-wave region where, in addition, monolithic waferscale integration of the source array should become possible.

\section{Other Integrated Antennas}

In addition to the integrated transmit and receive antennas discussed in the preceding sections, a number of other integrated mm-wave antennas has been developed in recent years for different applications. Examples include a beam steering reflector for the $94-\mathrm{GHz}$ band designed by Rutledge, Luhman, and their associates [54], [55] and a frequency multiplying slot array developed by the Itoh group [57].

The first antenna, sketched in Fig. 11(a), consists of a

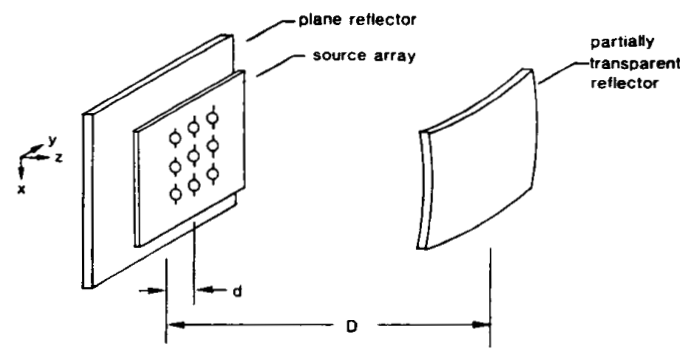

Fig. 10. Quasi-optical power combiner using Gaussian wavebeam resonator. The sources are distributed over one of the reflectors and are assumed to consist each of an active device such as an IMPATT diode integrated with a short dipole (from Mink [50]).

planar reflector, having a surface impedance that can be controlled electronically across the reflector surface. The essential part of the device is a square mesh of diode-loaded metal strips printed on a GaAs layer; see Fig. 11(b). The diodes are located on the vertical strips; they are Schottky type varactor diodes that are monolithically implanted in the GaAs substrate and, electrically, are in series with the vertical strips. The tangential component of the $E$ vector of the incident field is assumed to be parallel to the vertical strips of the grid which provide an inductive surface reactance (assuming that the GaAs substrate is a quarter wavelength (in GaAs) thick and is placed over a metal reflector). The capacitance of the varactor diodes is in series with and subtracts from this reactance. The diodes are controlled with the help of biasing voltages applied to the horizontal strips of the mesh, and the capacitance of each row of diodes can be adjusted independently. In this way the surface impedance of the reflector may be varied in the vertical direction and a progressive phase shift introduced in the reflected wave. Thus electronic beam steering becomes possible in the plane normal to the horizontal lines. A cross section view of a beam steering reflector using two such diode grids is shown in Fig. 11(c). The quartz cover serves as an antireflection layer; the metal ground plane acts as reflector, mounting block, and heat sink. The use of two grids instead of one, at a quarter wavelength spacing (where the impedance of one grid is inverted at the position of the other), has the advantage that a wide phase variation, over the entire region from $0^{\circ}$ to $360^{\circ}$, can be accomplished by varying the diode capacitances over a convenient finite range only. Hence, electronic beam steering over a wide angular range becomes possible.

Diode grids of the same type as shown in Fig. 11-but with the metal ground plane removed-can be utilized also as frequency multipliers, provided that the diode grid is biased so that the varactor diodes operate as nonlinear devices, and that the input and output signals are properly filtered. A multiplier of this type providing second harmonic generation of a $66-\mathrm{GHz}$ signal has been demonstrated by the Rutledge group [56]. The core of the device is a monolithic grid of 1600 Schottky varactor diodes fabricated on a $2-\mathrm{cm}^{2}$ GaAs wafer. 


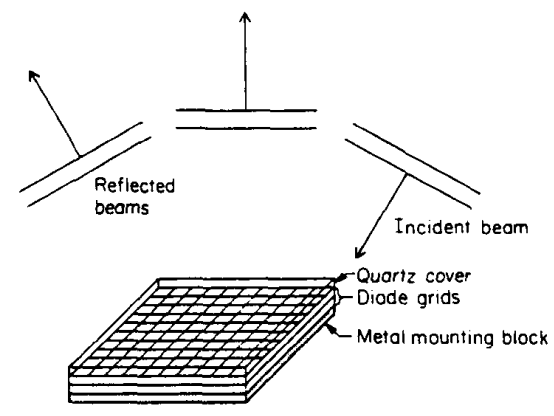

(a)

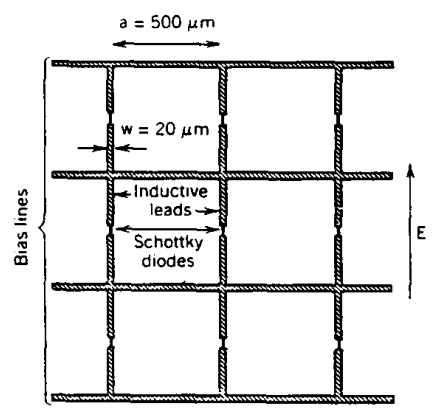

(b)

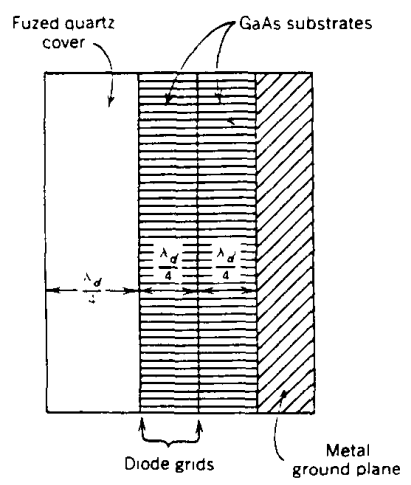

(c)

Fig. 11. Reflector antenna for electronic beam steering. (a) Configuration. (b) Square mesh of diode-loaded metal strips printed on GaAs substrate. The mesh dimensions apply to the $94-\mathrm{GHz}$ band. (c) Cross section of reflector (from Lam et al. [54]).

Using a different configuration, a diode-loaded printed slot array, the Itoh group [57] has developed an integrated frequency-multiplying transmit antenna. The radiating slots of this array are fed at the fundamental frequency where they are electrically short and provide little radiation. But they are a resonance and radiate strongly at the second harmonic generated by the diodes bridging the slots. The array in effect combines the functions of a distributed frequency multiplier, a filter, a free-space combiner, and an antenna in one integrated device. Fed by a microstrip line (that is printed on the backside of the antenna substrate), the array is a planar device that is well suited for beam shaping. The concept is interesting and likely to find numerous mm-wave applications.

\section{REFERENCES}

[1] D. N. Black and J. C. Wiltse, "Millimeter wave characteristics of phase-correcting Fresnel Zone Plates," IEEE Trans. Microwave Theory Tech., vol. MTT-35, pp. 1122-1128, Dec. 1987.

[2] J. C. Wiltse and J. E. Garrett, "The Fresnel Zone plate antenna," Microwave J., vol. 34, pp. 101-114, Jan. 1991.

[3] J. J. Lee, "Dielectric lens shaping and coma-correction zoning Part I: Analysis," IEEE Trans. Antennas Propagat., vol. AP-31, pp. 211-216, Jan. 1983.

[4] J. J. Lee and R. L. Carlise, "A coma-corrected multibeam shaped lens antenna, Part II: Experiments," IEEE Trans. Antennas Propagat., vol. AP-31, pp. 216-220, Jan. 1983.

[5] W. Rotman, "EHF dielectric lens antenna for multibeam MILSATCOM applications," in Dig. 1982 Int. IEEE-APS/URSI Symp., Albuquerque, NM, June 1982, pp. 132-135.

[6] E. C. Dufort and J. Uyeda, "A wide-angle scanning optical antenna," IEEE Trans. Antennas Propagat., vol. AP-31, pp. 60-67, Jan. 1983

[7] K. lizuka, M. Mizusawa, S. Urasaki, and J. Ushigome, "Volumetype holographic antenna," IEEE Trans. Antennas Propagat., vol. AP-23, pp. 807-810, Nov. 1975

[8] F. K. Schwering and A. A. Oliner, "Millimeter wave antennas," in Antenna Handbook, Y. T. Lo and S. W. Lee, Eds. New York: Van Nostrand Reinhold, 1988, ch. 17

[9] "Millimeter wave antennas," in Handbook of Microwave and Optical Components, K. Chang, Ed. New York: Wiley, 1988 , ch. 12.2

[10] J. W. Mink and F. K. Schwering, "Integrated Antennas," in Handbook of Microwave and Optical Components, K. Chang Ed. New York: Wiley, 1988, ch. 12.3 .

[11] P. Bhartia and I. J. Bahl, Millimeter Wave Engineering and Applications. New York: Wiley, 1984, ch. 9.

[12] P. Bhartia, K. V.S Rao, and R. S. Tomar, Millimeter-Wave Mi crostrip and Printed Circuit Antennas. Boston, MA/London, UK: Artech House, 1991.

[13] K. L. Klohn, R. E. Horn, H. Jacobs, and E. Freibergs, "Silicon waveguide frequency scanning linear array antenna," IEEE Trans. Microwave Theory Tech., vol. MTT-26, pp. 764-773, Oct. 1978.

[14] T. Itoh and B. Adelseck, "Trapped image guide leaky-wave antennas for millimeter wave applications," IEEE Trans. Antennas Propagat., vol. AP-30, pp. 505-509, May 1982

[15] F. Schwering and S. T. Peng, "Design of dielectric grating antennas for millimeter wave applications," IEEE Trans. Microwave Theory Tech., vol. MTT-31, pp. 199-209, Feb. 1983.

[16] M. Guglielmi and A. A. Oliner, "A practical theory for image guide leaky-wave antennas loaded by periodic metal strips," in Proc. 17th European Microwave Conf., Rome, Italy, Sept. $11-17,1987$, pp. 549-554.

[17] H. Maheri, M. Tsutsumi, and N. Kumagai, "Experimental studies of magnetically scannable leaky-wave antennas having a corrugated ferrite slab/dielectric structure," IEEE Trans. Antennas Propagat., vol. 36, pp. 911-917, July 1988.

[18] A. A. Oliner, "Scannable millimeter wave arrays," Weber Res. Inst., Polytechnic University, Tech. Rep. Poly-WRI-1543-88, vols. I and II, Sept. 30, 1988.

[19] K. S. Yngvesson, D. H. Schaubert, T. L. Korzeniowski, E. I. Kollberg, T. Thungren, and J. F. Johansson, "Endfire tapered slot antennas on dielectric substrates," IEEE Trans. Antennas Propagat., vol. AP-33, pp. 1392-1400, Dec. 1985.

[20] K. S. Yngvesson et al., "The tapered slot antenna-A new integrated element for millimeter-wave applications," IEEE Trans. Microwave Theory Tech., vol. 37, pp. 365-374, Feb. Trans.

[21] W. Menzel, "A new traveling wave antenna in microstrip," Arch. Electron. Ubertragung., vol. 33, pp. 137-140, Apr. 1979.

[22] J A Encinar, M. Guglielmi, and A. A. Oliner, "Taper optimization for sidelobe control in millimeter-wave metal-strip-loaded dielectric antennas," presented at the National Radio Science Meeting, Syracuse, NY, June 6-10, 1988. 
[23] M. A. Weiss, "Microstrip antennas for millimeter waves," IEEE Trans. Antennas Propagat, vol AP-29, pp 171-174 Jan. 1981.

[24] D. M. Pozar and D. H. Schaubert, "Comparison of architectures for monolithic phased array antennas," Microwave J., vol. 29, pp. 93-104, Mar. 1986.

[25] J. R. James and A. Henderson, "A critical review of millimeter planar arrays for military applications," presented at the Military Microwave Conf., London, U.K., Oct. 20-22, 1982.

[26] J. R. James and C. M. Hall, "investigation of new concepts for designing millimeter-wave antennas, Final Tech. Rep. on Contract DAJA37-80-C-0183, U.S. Army European Res. Office, Sept. 1983.

[27] E. Levine et al., "A study of microstrip array antennas with the feed network," IEEE Trans. Antennas Propagat., vol. 37, pp. 426-434, Apr. 1989.

[28] N. G. Alexopoulos, P. B. Katehi, and D. B. Rutledge, "Substrate optimization for integrated circuit antennas," IEEE Trans. Microwave Theory Tech., vol. MTT-31, pp. 550-557, July 1983

[29] D. M. Pozar, "Considerations for millimeter wave printed antennas," IEEE Trans. Antennas Propagat., vol. AP-31, pp. 740-747, Sept. 1983.

[30] Z. Rav-Noy, C. Zah, U. Schreter, D. B. Rutledge, T. C. Wang, S. E. Schwarz, and T. F. Kuech, "Monolithic Schottky diode imaging arrays at $94 \mathrm{GHz}$," in Dig. Infrared and Millimeter Wave Conf., Miami Beach, FL, Dec. 1983.

[31] H. R. Fetterman, T. C. L. G. Sollner, P. T. Parrish, C. D. Parker, R. H. Matthews, and P. E. Tannenwald, "Printed dipole millimeter wave antenna for imaging array applications," Electromagnetics, vol. 3, pp. 209-215, 1983.

[32] P. B. Katehi and N. G. Alexopoulos, "On the modeling of electromagnetically coupled microstrip antennas-The printed strip dipole," IEEE Trans. Antennas Propagat., vol. AP-32, pp. 1179-1186, Nov. 1984

[33] P. B. Katehi, N. G. Alexopoulos, and I. Y. Hsia, "A bandwidth enhancement method for microstrip antennas," IEEE Trans. Antennas Propagat., vol. AP-35, pp. 5-12, Jan. 1987.

[34] N. G. Alexopoulos and D. R. Jackson, "Fundamental superstrate (cover) effects on printed circuit antennas," IEEE Trans. Antennas Propagat., vol. AP-32, pp. 807-816, Aug. 1984.

[35] H. Y. Yang and N. G. Alexopoulos, "Generation of nearly hemispherical and high gain azimuthally symmetric patterns with printed circuit antennas," IEEE Trans. Antennas Propagat., vol. AP-35, pp. 972-977, Aug. 1987.

[36] _ "Gain enhancement methods for printed circuit antennas through multiple superstrates," IEEE Trans. Antennas Propagat., vol. AP-35, pp. 860-863, July 1987.

[37] D. R. Jackson and A. A. Oliner, "A leaky-wave analysis of the high-gain printed antenna configuration," IEEE Trans. Antennas Propagat., vol. 36, pp. 905-910, July 1988.

[38] T. Itoh, "Recent progress of quasi-optical integrated microwave and millimeter-wave circuits and components," Alta Freq., vol. LVIII, no. 5-6, pp. 71-79, Sept.-Dec. 1989.

[39] D. B. Rutledge, D. P. Neikirk, and D. P. Kasilingam, "Integrated circuit antennas," in Infrared and Millimeter Waves, K. J. Button, Ed. Orlando, FL: Academic, vol. 10, 1983, ch. . 1.

[40] R. J. Mailloux, "Array elements and architecture of printed circuit array antennas," IEEE MTT-S Newsletter, pp. 29-32, 1988.

[41] J. A. Kinzel, "GaAs technology for millimeter wave phased arrays," IEEE AP-S Newsletter, pp. 12-14, Feb. 1987.

[42] J. A. Kinzel, "Recent Advances in Monolithic Millimeter Wave Arrays," in Dig. 1990 Int. IEEE-APS/URSI Symp., Dallas, TX, vol. IV, pp. 1402-1405, May 1990.

[43] K. D. Stephan, N. Camilleri, and T. Itoh, "A quasi-optical polarization duplexed balanced mixer for mm-wave applications," IEEE Trans. Microwave Theory Tech., vol. MTT-31, pp. 164-170, Feb. 1983; Also, "Erratum", vol. MTT-31, p. 504, June 1983.

[44] C. Zah et al., "Millimeter wave monolithic schottky diode imaging arrays," Int. J. Infrared and mm-Waves, vol. 6, pp.
981-997, Oct. 1985.

[45] G. M Rebeiz et al, "Monolithic millimeter-wave twodimensional horn imaging arrays," IEEE Trans. Antennas Propagat., vol. 38, pp. 1473-1482, Sept. 1990.

[46] W. Y. Ali-Ahmad, et al., "94 GHz integrated-horn antennas: Impedance, pattern and double polarized applications," in Dig. 15th Int. Conf. Infrared and Millimeter-Waves, Orlando, FL, Dec. 10-14, 1990, pp. 614-619.

[47] N. Camilleri and B. Bayrakaroglu, "Monolithic millimeter-wave IMPATT oscillator and active antenna," IEEE Trans. Microwave Theory Tech., vol. 36, pp. 1670-1676, Dec. 1988.

[48] J. Birkeland and T. Itoh, "FET-based planar circuits for quasioptical sources and transceivers," IEEE Trans. Microwave Theory Tech, vol. 37, pp. 1452-1459, Sept. 1989.

[49] B. S. Song and T. Itoh, "Distributed Bragg reflection dielectric waveguide oscillators," IEEE Trans. Microwave Theory Tech., vol. MTT-27, pp. 1019-1025, Dec. 1979.

[50] J. W. Mink, "Quasi-optic power combining of solid-state millimeter-wave sources," IEEE Trans. Microwave Theory Tech., vol. MTT-34, pp. 273-279, Feb. 1986.

[51] R. A. York and R. C. Compton, "A $4 \times 4$ active array using Gunn diodes," Digest of 1990 Int. IEEE-APS/URSI Symp., Dallas, TX, vol. III, pp. 1146-1149, May 1990.

[52] R. M. Weikle et al., "A 100-element MESFET grid oscillator," Digest of 1990 Int. IEEE-APS/URSI Symp., Dallas, TX, vol. III, pp. 1174-1177, May 1990.

[53] D. B. Rutledge et al., "Quasi-optical power-combining arrays," Digest of 1990 IEEE MTT-S Int. Microwave Symp., Dallas, TX, vol. III, pp. 1201-1204, May 1990.

[54] W. W. Lam et al., "Diode grids for electronic beam steering and frequency multiplication," Int. J. Infrared and Millimeter Waves, vol. 7, pp. 27-41, Jan. 1986.

[55] W. W. Lam et al., "Millimeter-wave diode-grid phaseshifter," IEEE Trans. Microwave Theory Tech., vol. MTT-36, pp. 902-907, May 1988.

[56] C. F. Jou et al., "Millimeter-wave diode-grid frequency doubler," IEEE Trans. Microwave Theory Tech., vol. MTT-36, pp. 1507-1514, Nov. 1988.

[57] S. Nam, T. Uvano, and T. Itoh, "Microstrip-fed planar frequency-multiplying space combiner," IEEE Trans. Microwave Theory Tech., vol. MTT-35, pp. 1271-1276, Dec. 1987.

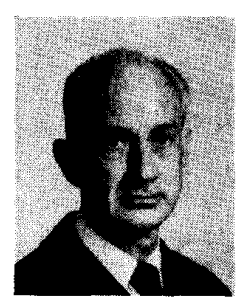

Felix K. Schwering (Fellow, IEEE) was born on June 4, 1930, in Cologne, Germany. He received the Dipl. Ing. degree in electrical engineering and the Ph.D. degree from the Technical University of Aachen, West Germany, in 1954 and 1957, respectively.

From 1956 to 1958 he was Assistant Professor at the Technical University of Aachen. In 1958 he joined the U.S. Army Research and Development Laboratory at Fort Monmouth, NJ, where he performed basic research in free space and guided propagation of electromagnetic waves. From 1961 to 1964 he worked as a member of the Research Staff of the Telefunken Company, Ulm, Germany, on radar propagation studies and missile electronics. In 1964 he returned to the U.S. Army Electronics Command, Fort Monmouth, $\mathrm{NJ}$, and has since been active in the fields of electromagnetic-wave propagation, diffraction and scatter theory, theoretical optics, and antenna theory. Recently, he has been involved, in particular, in mm-wave antenna and propagation studies. He is a Visiting Professor at New Jersey Institute of Technology, Newark, NJ.

Dr. Schwering is a member of URSI, Commission B. He received the 1961 and 1982 Best Paper Awards of the IEEE Antennas and Propagation Society (jointly with G. Goubau), and from 1987 to 1989 served as a Distinguished Lecturer of this society. 